\title{
Childhood sclerosing cholangitis associations in a Tunisian tertiary care hospital: a many-faceted disease
}

\author{
Miniar Tfifha ${ }^{1}$, Tarek Kamoun², Nadia Mama², Sarra Mestiri ${ }^{3}$ Saida Hassayoun ${ }^{1}$, \\ Noura Zouari ${ }^{1}$, Hela Jemni ${ }^{2}$, Saoussen Abroug ${ }^{1}$ \\ Departments of ${ }^{1}$ Pediatric and ${ }^{2}$ Radiology Sahloul Hospital; ${ }^{3}$ Department of Anatomopathology, Farhat Hached Hospital, \\ Sousse, Tunisia. E-mail: miniartfifha@yahoo.fr \\ Received: 27th May 2017, Revised: 16th July 2017, Accepted: 13th August 2017
}

SUMMARY: Tfifha M, Kamoun T, Mama N, Mestiri S, Hassayoun S, Zouari $\mathrm{N}$, Jemni $\mathrm{H}$, Abroug $\mathrm{S}$. Childhood sclerosing cholangitis associations in a Tunisian tertiary care hospital: a many-faceted disease. Turk J Pediatr 2019; 61: 905-914.

Sclerosing cholangitis (SC) is a liver disorder affecting children and adults, causing chronic cholestasis and secondary biliary cirrhosis. The purpose of this study was to present different associated diseases to SC in a Tunisian tertiary care hospital.

Six patients were identified with SC associated with other diseases, four males and two females. The first symptom was liver enlargement in all cases with abnormal liver biochemistry. A moderate increase in AST and ALT levels was registered in all cases with moderate cholestasis in 4 patients. Three of them presented an auto-immune condition. Two patients were diagnosed with auto-immune hepatitis prior to SC and Crohn disease in only one patient. One developed linear IgA bullous dermatosis. Three patients were diagnosed with Multisystemic Langerhans Cell Histiocytosis (LCH). The primary site of LCH was the liver associated secondary to insipidus diabetes (one case), mastoiditis (two cases) and chest localization (one case). The outcome of those patients was variable with poor prognosis especially for SC secondary to $\mathrm{LCH}$. No patient underwent liver transplantation.

$\mathrm{SC}$ is a rare disorder with variable clinical presentations. To our knowledge, this is the first report of this condition in Tunisian and North African children. Diagnosis and treatment of SC and its associations remains a challenge, especially because there is still no effective medical therapy aimed at preventing disease progression. Pediatric liver transplantation is the only life-extending therapeutic alternative for patients with end-stage liver failure. Liver transplantation has not been performed on young children in our country.

Key words: sclerosing cholangitis, childhood, cholestasis, langerhans histiocytosis.

Sclerosing cholangitis (SC) is a rare cause of chronic cholestasis and secondary biliary cirrhosis in children, which is characterized by inflammation and obliterative fibrosis of the intrahepatic and/or extrahepatic bile ducts. ${ }^{1}$ Five main clinical forms are described: Neonatal sclerosing cholangitis (NN-SC), SC with autoimmune features, SC secondary to various diseases, including Langerhans cell histiocytosis (LCH) and immunodeficiencies (ID) and SC without autoimmune features and unknown etiology. ${ }^{1,2}$ Only in those pediatric patients in whom SC occurs without any of the previously mentioned defining features should the name "primary" be used. ${ }^{3}$

Even though childhood SC has been extensively investigated in the West, data regarding the disease in southern regions is lacking. Studies on SC are relatively sparse and most publications are single case reports in North Africa. What makes the image more 
blurred is the existence of marked variability in the clinical manifestations and associated conditions. To our knowledge, this is the first report of this condition in Tunisia. We thus reported a single center case series concerning SC associations to improve the understanding of this disease with various entities.

\section{Material and Methods}

The current study was conducted to investigate the situation of childhood SC associated with other diseases in the Sahloul pediatric department, a tertiary care hospital, and to compare it with other studies worldwide.

\section{Patients and laboratory methods}

We reviewed the pediatric cases diagnosed with SC, from May 2012 to June 2016, in the pediatric department at the Sahloul University Hospital in Sousse, Tunisia. The clinical, laboratory, radiologic and histopathological findings, responses to treatment and evolution are reported.

Biochemical tests: Alanine Aminotransferase (ALT), Aspartate Aminotransferase (AST), gamma - glutamyl transpeptidase (GGT) and bilirubin were measured by an automated chemical analyzer (Unicel DxC 660i synchronBeckman Coulter). All the laboratory methods used had positive and negative controls, internal controls and instruments calibration.

\section{Radiologic data}

Abdominal ultrasonography (US) was done in all cases. Magnetic resonance (MR) cholangiography, in two cases were explored by a Philips Ingenia 1.5T MRI system machine and the other 4 by a 1.5 Tesla GE machine. Other radiologic data were reported (chest CT in one patient, brain CT in three patients and brain MR imaging in two patients).

Histopathology tests obtained by liver biopsies were reported using histopathological criteria described elsewhere.

The study was approved by the ethical committee of the institutional noninterventional clinical studies of Sahloul.

\section{Results}

Six Tunisian children, four males and two females were diagnosed with SC during the studied period. Their ages at presentation ranged from 1 to 8 years (median 4 years). Clinical features including presenting signs and symptoms, associated diseases, and response to therapy are summarized in Table I.

The liver involvement was initially discovered by liver enlargement confirmed on abdominal US in all cases with abnormal liver biochemistry (Table I). A moderate increase in AST and ALT levels were registered in all cases, with moderate cholestasis in five patients. MR imaging and other radiologic findings as well as liver biopsy results are presented in Table II.

Two patients had autoimmune hepatitis (AIH) associated to SC with Crohn's disease $(C D)$ in only one case. Initial liver biopsies were consistent with $\mathrm{AIH}$ (intense interface hepatitis, with an abundant lymphoplasmocytic infiltrate, giant cell transformation of hepatocytes and bridging fibrosis), but MR cholangiography changes (Fig. 1) and follow-up liver biopsies were suggestive of SC (inflammatory destruction of biliary structures and lobular cholestasis) (Fig. 2). These patients received Tacrolimus therapy with Ursodeoxycholic acid (UDCA). It successfully controlled the disease.

After one year, one of the patients with SC developed a linear IgA bullous dermatosis (LABD) confirmed by both histological and immunofluorescence studies. Dapsone was the first-line therapy, in this case with prompt response. However, fatal bleeding oesophageal varices caused death at age 9 .

Three of our patients had biliary cirrhosis with SC secondary to LCH (Fig. 3). Among other extra hepatic manifestations, radiological studies showed osteolytic and pulmonary lesions (Fig. 4 and 5). Central diabetes insipidus (CDI) was diagnosed in one patient (patient 5) by excessive urination and extreme thirst associated with pituitary abnormalities (Fig. 6). The liver wedge biopsy reported typical granulomatous lesions with positivity of the immunostain by the antibody anti PS100 on the histiocyte membranes in only one patient 


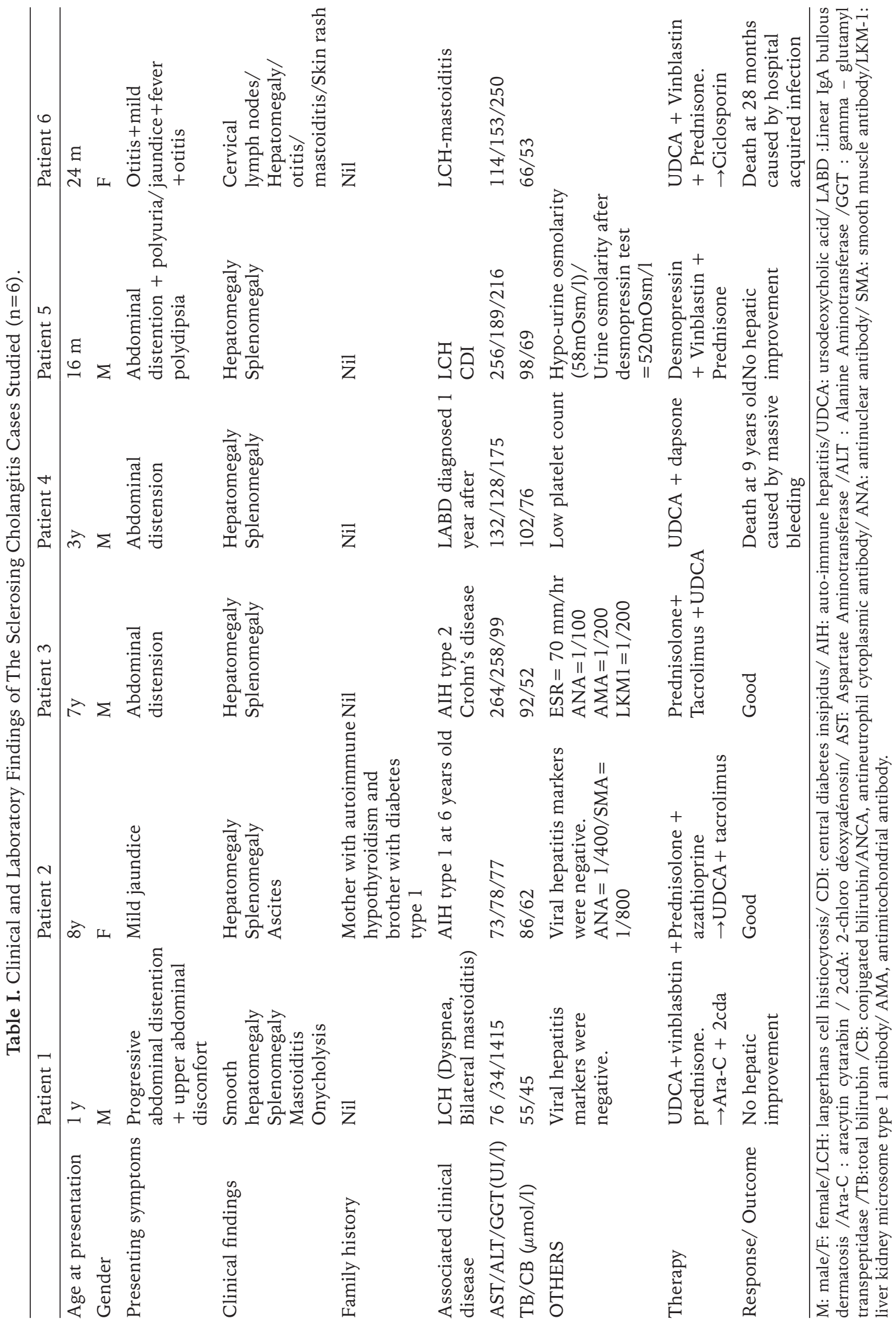


Table II. Imaging and Histopathological Findings at Diagnosis of Sclerosing Cholangitis $(n=6)$.

\begin{tabular}{llll}
\hline & MR Cholangiography & Liver biopsy histopathology & Other interesting findings \\
\hline Patient 1 & - Vanishing bile duct & Interstitial fibrosis and inflammatory & - interstitial pneumonia \\
& syndrome & infiltrates in the portal area-Epithelial & - bilateral mastoiditis \\
& - Periductal fibrosis. & injury and reactive hyperplasia of small & (positive immunostaining \\
& - Dysmorphic liver & bile ducts with cholestatic features- & for CD1a on temporal \\
& Histiocyte aggregation and lipid & lesion biopsy) \\
& granuloma formation-negative CDla &
\end{tabular}

Patient 2 -Segmental intra hepatic bile duct dilatation and stenosis. -Proximal strictures of the right and left hepatic ducts.

Patient 3 Periductal fibrosis Dysmorphic liver

Patient 4 Non-contributory

Patient 5 - Segmental intrahepatic bile duct dilatation and stenosis.

- Proximal strictures of the right and left hepatic ducts.

Patient 6 Segmental intra hepatic bile duct dilatation and stenosis.

- Periductal fibrosis.

- Dysmorphic liver.

\section{immunostain}

Bile duct damage around the portal area-Focal duct obliteration by a loose concentric fibrosis-Lympho-plasmocytic

infiltration located predominantly around the perilobular areas.

First biopsy: Dense portal lymphoplasmocytic cell infiltrate with marked Interface hepatitis-Second biopsy: inflammatory destruction of biliary structures and lobular cholestasis

Portal spaces enlarged by portal fibrosis with extensive bridging, displaying a cirrhotic pattern-Portal inflammatory lymphocytic infiltrate-Lymphocytic cholangitis and periductal fibrosis.

\section{Nil} - Colonoscopy showed
scattered reddened mucosa with small aphtoid ulcers in the caecum and transverse colon.

- Direct

Immunofluorescence of skin biopsies showed linear deposits of IgA along the dermoepidermal junction

Micronodular cirrhosis-Bile plugs-Paucity Absence of the posterior of intrahepatic bile ducts-Positive PS100 pituitary bright spot on immunostain MRI.

- Portal spaces enlarged by fibrosis and - interstitial pneumonia an inflammatory infiltrate (lymphocytes, left mastoiditis (positive histiocytes with grooved nuclei and immunostaining for eosinophils-Biliary ducts infiltrated by $\mathrm{CDla}$ on temporal lesions lymphocytes and surrounded by a loose Biopsy) concentric fibrosis. (patient 5) (Fig. 7). The two other liver biopsies showed portal lesions with bile duct damages, ductular proliferation and portal fibrosis, related to multifocal infiltration of liver by Langerhans cell histiocytes. The diagnosis of LCH was confirmed on the lytic lesion of mastoid bone with immunohistochemistry showing positivity for both CD1a and PS100.

Variable responses to conventional treatment were observed with a poor prognosis for SC secondary to $\mathrm{LCH}$ despite conventional chemotherapy based on vinblastin and steroids in all cases.
Written informed consent was obtained from the patients' parents for publication of the cases and any accompanying images.

\section{Discussion}

SC may complicate a wide variety of disorders, including primary and secondary immunodeficiencies, LCH, psoriasis, cystic fibrosis, reticulum cell sarcoma, and sickle cell anemia. Moreover, an overlap syndrome between $\mathrm{AIH}$ and SC is significantly more common in children than in adults. ${ }^{3}$ There is a limited number of published series of pediatric SC, especially in North Africa. The 


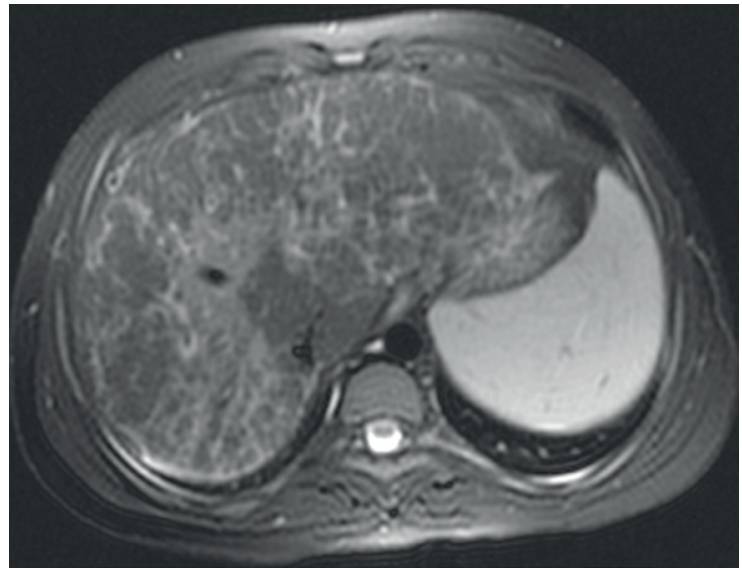

Fig. 1. Axial T2 fat sat MR imaging of the liver: high signal intensity of intra hepatic linear septation consisting with fibrosis

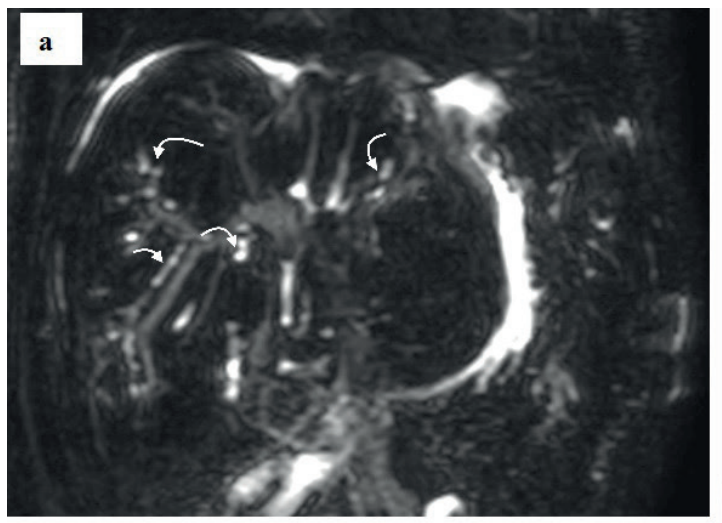

Fig. 3. Typical pattern of sclerosing cholangitis: MRCP with MIP 5mm slice image and postcontrast T1 image: several images of intra hepatic bile duct dilatation and stenosis (white arrows). Peri portal enhancement consistent with fibrosis (black arrows)

disease's specificities in Tunisia are unknown particularly with the associated conditions.

Childhood SC affects mostly boys with an onset in the second decade of life. ${ }^{4}$ In our case series, the majority are boys (Sex ratio= $4 / 2$ ) and all patients were affected in the first decade of life. However, in recent publications, similar proportion of boys and girls is reported in autoimmune SC. ${ }^{2}$

Most patients presented with symptoms and/ or signs of chronic liver disease. ${ }^{4}$ Hepatomegaly

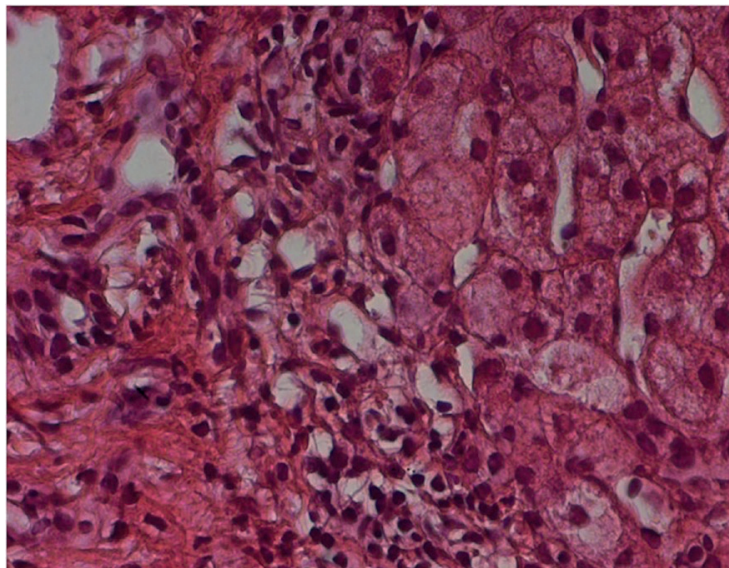

Fig. 2. H.E x 400: Severe interface hepatitis, made of predominantly lympho-plasmocytic inflammatory infiltrate, associated to lymph-epithelial lesions of biliary ducts, inflammatory destruction of biliary structures and lobular cholestasis.

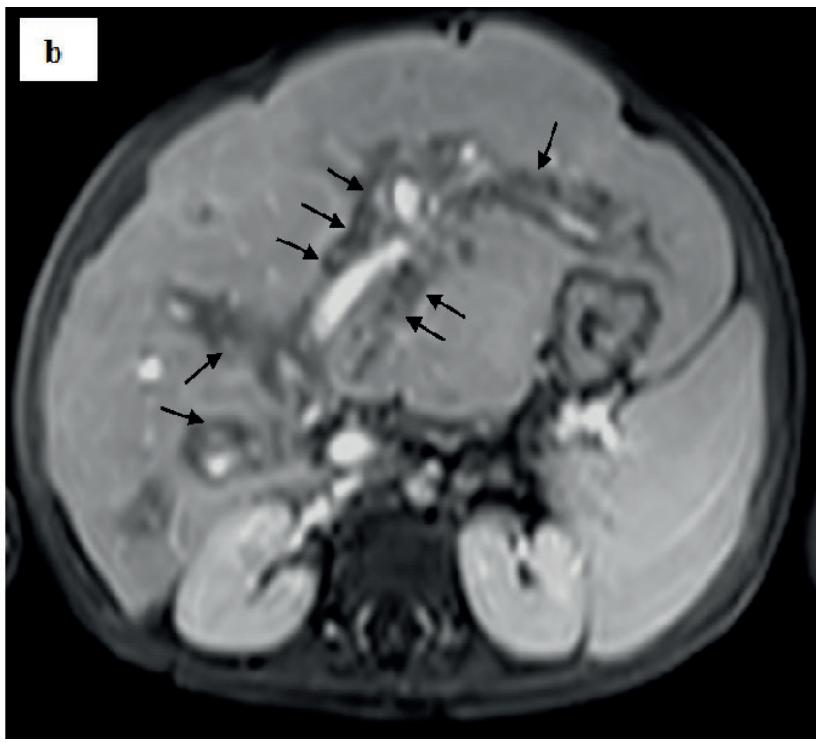

is a key sign of liver involvement, along with other hepatic signs and symptoms indicate the need for further investigation. ${ }^{5}$ Cholestasis with abnormal liver enzymes is the hallmark of SC but cannot be distinctive for clinical features leading to differentiation between hepatocellular and obstructive disorders. ${ }^{6}$

In this case series, the two most frequent clinical forms of childhood SC were reported: SC with autoimmune features (associated with $\mathrm{AIH}, \mathrm{CD}$ and LABD) and SC secondary to 


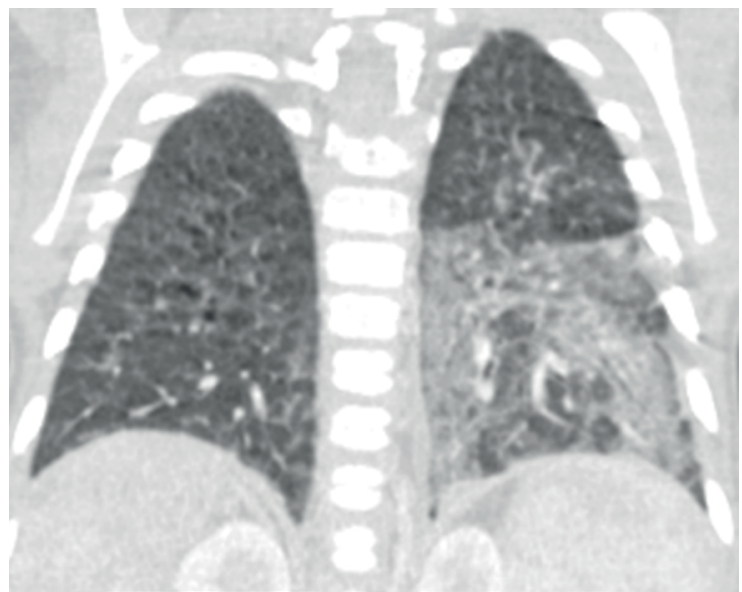

Fig. 4. Pulmonary CT scan: coronal MPR thin slice image: ground glass of the inferior lobe opacity with septal thickening.
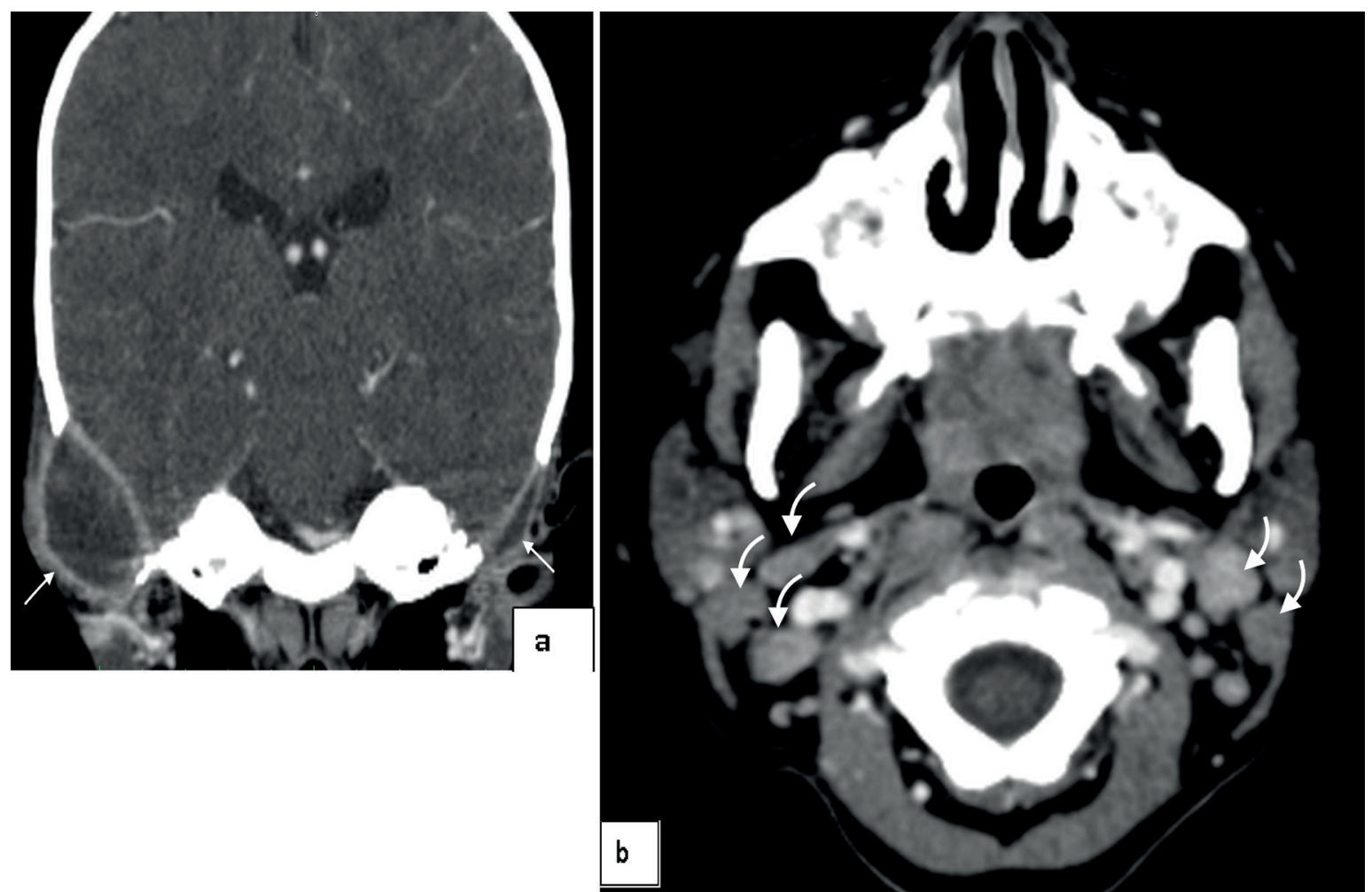

Fig. 5. Brain CT scan: Enhanced coronal (a) and axial (b) MPR thin slice images: bilateral temporal bone osteolysis and mastoid collections with peripheral enhancement (straight arrows). Bilateral cervical adenomegalies (curved arrows).

LCH. ${ }^{7-9}$ MR Cholangiography findings showed morphologic information and contribute to the definitive diagnosis in the majority of our cases. At onset or in the following years, lesions of larger bile ducts with duct wall irregularities, strictures, dilations, and beading result in the characteristic "beadon-a-string" appearance. ${ }^{10,11}$ Some difficulties can be encountered in performing the MR Cholangiography, particularly in obtaining apnea in non-cooperative young patients, and especially in the absence of general anesthetic. 


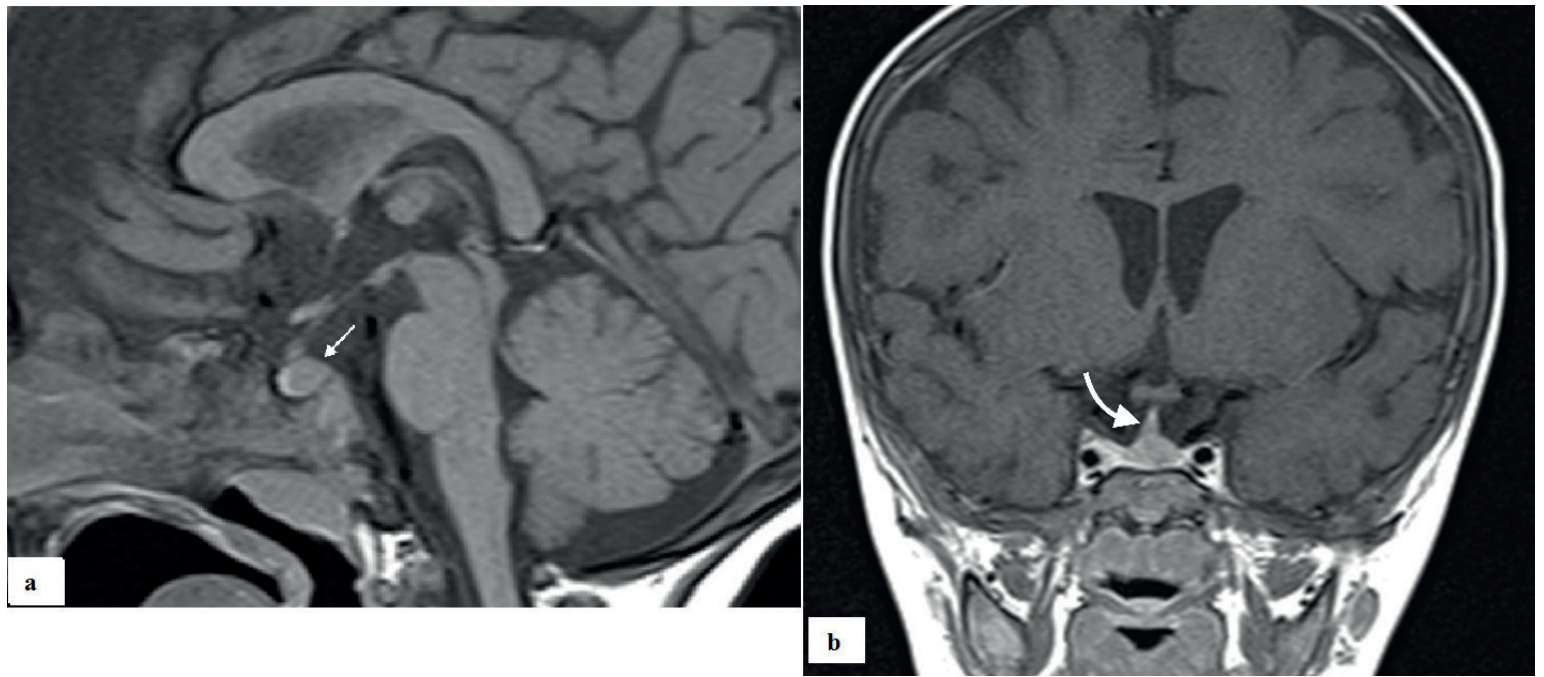

Fig. 6. MR imaging of the pituitary region. a: SET1 sagittal image, b: Post contrast T1 coronal image: lack of the normal T1 high signal intensity of the posterior pituitary. Abnormal nodular thickening of the pituitary stalk (arrow).

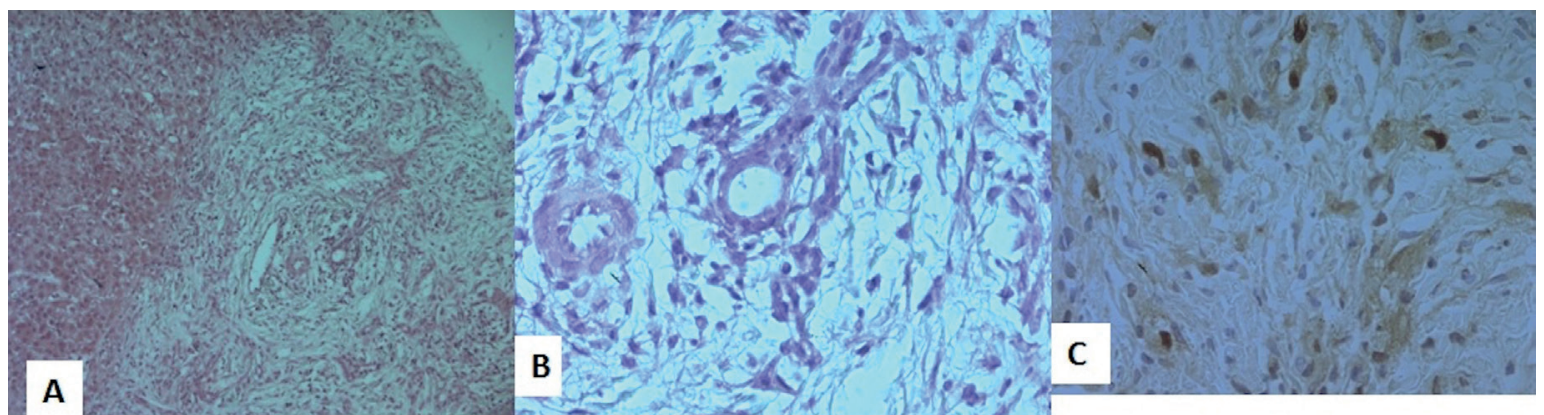

Fig. 7. Liver biopsy (A) H.E x 100 : Portal spaces containing a loose peri-biliary concentric fibrosis, associated to neo-ductules proliferation and to a mononuclear inflammatory infiltrate, containing histiocytes (B) H.E $\mathrm{x}$ 400: Biliary ductules are lined by a dystrophic epithelium, associated to lymphocytic exocytosis (C) IHC x 400: Intense immunostaining of histiocytes with PS100.

It was the case of two of our patients. In those cases, the diagnosis was based on the clinical history, laboratory and histological data. ${ }^{1,5}$

\section{Autoimmune hepatitis - Sclerosing Cholangitis}

This entity, named AIH/SC overlap syndrome or autoimmune sclerosing cholangitis (ASC), was first described by Maggiore et al. ${ }^{12}$ Pediatric-onset of ASC is rare. ${ }^{13}$ It is well individualized as a clinical form of SC in childhood, characterized by the association of $\mathrm{AIH}$ and SC, occurring either simultaneously or within a short interval. ${ }^{2}$ For the 2 patients reported in this study, the diagnosis of overlap syndrome was made respectively 2 and 4 years after the onset of the AIH. Evolution from $\mathrm{AIH}$ to SC has been documented suggesting that $\mathrm{AIH}$ and SC are part of the same pathogenic process involving hepatocytes in $\mathrm{AIH}$ and bile ducts in SC. ${ }^{2}$ The clinical onset of ASC for our patients was similar to that of AIH. Standard liver function tests were not helpful in discriminating between AIH and ASC. It is reported that the alkaline phosphatase/AST ratio is significantly higher in ASC. ${ }^{2}$ Diagnosis of AIH is supported by the histological finding of interface hepatitis with massive portal infiltration of mononuclear cells and plasmocytes. Inflammatory bile duct lesions are not unusual and may suggest features of "overlap" with ASC. ${ }^{10,11}$ 
At present, none of the patients included in the present case series had genetic studies performed, as genotyping is not required to render a diagnosis of AIH/ASC. However, consanguineous parentage was reported in the two patients with familiar history of immune mediated disorders (mother with autoimmune hypothyroidism and brother with diabetes type 1 in case 2), lending some weight to the hypothesis that a combination of one or more genes, combined with environmental factors, may play a role in the etiology of ASC.

In the current case series, the two patients with ASC only had intra-hepatic involvement. Feldstein et al. $^{4}$ found only intra-hepatic involvement in about $40 \%$ and only extrahepatic in $2 \%$ of the children.

ASC responds to the same immunosuppressive treatment used for AIH. ${ }^{14}$ Tacrolimus was started early in our patients, with resolution of liver test abnormalities within a few months. But its medium-to long-term prognosis is worse than that of AIH because of progression of bile duct disease towards biliary cirrhosis and chronic liver failure (in 17 to 21\%) despite treatment. ${ }^{4,15}$ UDCA is added to immunosuppressive treatment, but its role in cancelling the progression of the bile duct disease should be assessed. ${ }^{15}$

However, there is currently no effective treatment for patients with ASC, which could prevent gradual progression of liver disease to biliary cirrhosis and chronic liver failure. ${ }^{16}$ Liver transplantation in young children, not available until now in Tunisia, remains the only effective therapeutic option for patients with end-stage liver disease. ${ }^{1}$

As in adults, SC in children can be associated with IBD. ${ }^{1}$ Association of AIH-SC overlap syndrome with ulcerative colitis (UC) is well recognized but is rarely seen with $\mathrm{CD} \cdot{ }^{17,18} \mathrm{In}$ our case report, CD was diagnosed 2 years after the SC. Such association must be taken into consideration and in order to look for IBD, a colonoscopy must be performed in all patients with AIH-SC overlap syndrome regardless of the presence of symptoms of IBD. In fact, children with this liver condition frequently suffer from IBD, but the bowel disease may be silent and discovered only if an endoscopic procedure with biopsy is performed. ${ }^{4}$

As opposed to AIH and ASC, there are no definitive management guidelines for ASC whether it occurs by itself or in association with either of the two IBD subtypes. ${ }^{18}$ The current trend is to treat each disease entity separately and adjust medications according to symptoms and side effects. ${ }^{17} \mathrm{~A}$ beneficial effect of oral Vancomycin $(50 \mathrm{mg} / \mathrm{kg}$ per day) has been reported. ${ }^{19}$

\section{Sclerosing Cholangitis associated with $L A B D$ :}

LABD is a rare blistering disease characterized by pruritic subepidermal blisters. This entity was once considered a subset of dermatitis herpetiformis (DH) but has been reclassified since as a distinct entity based on the distinct linear deposition of IgA at the epidermal basement membrane. We describe an unusual case of LABD associated to SC with poor outcome. To the best of our knowledge, this is the second case reported with both LABD and SC and the first pediatric case. ${ }^{20}$

Ulcerative colitis, $\mathrm{CD}$ and gastric hypochlorhydria have been described in association with LABD. Evidence of the association of autoimmune disease with LABD is not sufficient to allow statistical analysis of prevalence, but there are reports of this disease in association with systemic lupus erythematosus, dermatomyositis, thyrotoxicosis, autoimmune haemolytic anaemia and rheumatoid arthritis. ${ }^{21}$ The association of SC and LABD raises the possibility of a common immunopathogenic mechanism involved in the development of both diseases.

Treatment was based on Dapsone $25 \mathrm{mg}$ daily for the LABD and UDCA for the SC in our case. It is of importance to note that there was no relation between our patient's LABD and the activity of the SC.

\section{SC and $\mathrm{LCH}$}

$\mathrm{LCH}$ has a variety of clinical features from mild illness to life-threatening multi-organic failure. 
Generally, liver involvement, relatively frequent especially in children younger than 5 years old, is a component of multisystem disease in $\mathrm{LCH}$ with extreme case-to-case variation. ${ }^{22,23}$ SC was found in 1.3-6\% of children with $\mathrm{LCH}$ in a multicenter retrospective study done in France. ${ }^{22}$ In our three cases, the diagnosis of SC was made before or at the same time of the diagnosis of LCH. Liver biopsy may demonstrate infiltration of the portal area and basement membrane of the bile ducts with Langerhans cells. Immunostaining for PS100 and CD1a cells is required for the definitive diagnosis. ${ }^{23}$

Only one liver specimen tested was positive for PS100. For the two others patients, the LCH was confirmed on the mastoid bone erosion biopsy. It can be explained by a high degree of cirrhosis at the diagnosis of LCH in our patients.

The systemic manifestations were also different in our cases: bone and lung involvement consisting in bitemporal bone erosion and reticular pulmonary opacities with diffuse small cysts in thoracic CT scan in patient 1 . Associated mastoid ostelysis was noted in patient 1 and 6 . In the third case, SC was associated to an involvement of the hypothalamo-pituitary axis (CDI) (patient 5). To the best of our knowledge, liver involvement associated with CDI in the same patient as a clue to the diagnosis of $\mathrm{LCH}$ was reported in only one case before in the literature review. ${ }^{24}$

On average, $\mathrm{SC}$ manifests 2 years after diagnosis of $\mathrm{LCH}$ in children. It is rarely indicative of the diagnosis, which is mainly based on radiological examinations. Liver involvement is a factor of poor prognosis. ${ }^{2,22,25}$ It precipitates the occurrence of biliary cirrhosis and liver failure. ${ }^{22}$ The treatment used in these 3 cases was vinblastine-prednisone association, which was recommended by the Histiocyte Society without any improvement. The same results were observed despite changing chemotherapy.

Pediatric liver transplantation, which was not available in our country, is the treatment of choice for liver failure to SC in $\mathrm{LCH} ., 25$

SC is a rare cause of chronic cholestasis and secondary biliary cirrhosis in children. The various clinical presentations in children and frequent extrahepatic associations are challenging. This case series is the first study regarding childhood SC in North Africa. The specificities are the singularity of SC associations, especially CD, CDI and LABD. This study is limited by a small number of patients in one pediatric center and its retrospective nature. Retrospective multicenter studies are required in Tunisia to identify the most relevant associations in pediatric SC in a population with high rate of consanguineous patients and genetic drift. ${ }^{26}$ Meanwhile liver transplantation, complications of advanced liver disease and portal hypertension need to be assessed and may decrease morbidity and mortality.

\section{REFERENCES}

1. Tenca A, Färkkilä M, Jalanko H, et al. Environmental risk factors of pediatric-onset primary sclerosing cholangitis and autoimmune hepatitis. J Pediatr Gastroenterol Nutr 2016; 62: 437-442.

2. Girard M, Franchi-Abella S, Lacaille F, Debray D Specificities of sclerosing cholangitis in childhood. Clin Res Hepatol Gastroenterol 2012; 36: 530-535.

3. Mieli-Vergani G, Vergani D. Sclerosing cholangitis in children and adolescents. Clin Liver Dis 2016; 20: 99 111.

4. Feldstein AE, Perrault J, El-Youssif M, Lindor KD, Freese DK, Angulo P. Primary sclerosing cholangitis in children: A long-term follow-up study. Hepatology 2003; 38: 210-217.

5. Karcaaltincaba M, Haliloglu M, Akpinar E, et al. Multidetector CT and MRI findings in periportal space pathologies. Eur J Radiol 2007; 61: 3-10.

6. Singh S, Talwalkar JA. Primary sclerosing cholangitis: diagnosis, prognosis and management. Clin Gastroenterol Hepatol 2013; 11: 898-907.

7. Debray D, Pariente D, Urvoas E, Hadchouel M, Bernard O. Sclerosing cholangitis in children. J Pediatr 1994; 124: 49-56.

8. Mieli-Vergani G, Vergani D. Sclerosing cholangitis in the paediatric patient. Best Pract Res Clin Gastroenterol 2001; 15: 681-690.

9. Miloh T, Arnon R, Shneider B, Suchy F, Kerkar N A retrospective single-center review of primary sclerosing cholangitis in children. Clin Gastroenterol Hepatol 2009; 7: 239-245.

10. Malik N, Venkatesh SK. Imaging of autoimmune hepatitis and overlap syndromes. Abdom Radiol (NY) 2017; 42: 19-27. 
11. Maggiore G, Riva S, Sciveres M. Autoimmune diseases of the liver and biliary tract and overlap syndromes in childhood. Minerva Gastroenterol Dietol 2009; 55: 53-70.

12. Maggiore G, Veber F, Bernard O, et al. Autoimmune hepatitis associated with anti-actin antibodies in children and adolescents. J Pediatr Gastroenterol Nutr 1993; 17: 376-381.

13. Deneau M, Jensen MK, Holmen J, Williams MS, Book LS, Guthery SL. Primary sclerosing cholangitis, autoimmune hepatitis, and overlap in Utah children: epidemiology and natural history. Hepatology 2013; 58: 1392-1400

14. Mieli-Vergani G, Vergani D. Autoimmune liver diseases in children - what is different from adulthood? Best Pract Res Clin Gastroenterol 2011; 25: 783-795.

15. Floreani A, Liberal R, Vergani D, Mieli-Vergani G. Autoimmune hepatitis: Contrasts and comparisons in children and adults-a comprehensive review. J Autoimmun 2013; 46: 7-16.

16. Smolka V, Karaskova E, Tkachyk O, et al. Long-term follow-up of children and adolescents with primary sclerosing cholangitis and autoimmune sclerosing cholangitis. Hepatobiliary Pancreat Dis Int 2016; 15: 412-418.

17. Malik TA, Gutierrez AM, McGuire B, Zarzour JG, Mukhtar F, Bloomer J. Autoimmune hepatitis-primary sclerosing cholangitis overlap syndrome complicated by Crohn's disease. Digestion 2010; 82: 24-26.

18. Saich R, Chapman R. Primary sclerosing cholangitis, autoimmune hepatitis and overlap syndromes in inflammatory bowel disease. World J Gastroenterol 2008; 14: 331-337.

19. Davies YK, Cox KM, Abdullah BA, Safta A, Terry AB, Cox KL. Long-term treatment of primary sclerosing cholangitis in children with oral vancomycin: an immunomodulating antibiotic. J Pediatr Gastroenterol Nutr 2008; 47: 61-67.
20. Hecker MS, Hecker DJ, Weinberg JM. Linear IgA bullous dermatosis in a patient with sclerosing cholangitis. J Eur Acad Dermatol Venereol 2002; 16: 83-84.

21. Tobón GJ, Toro CE, Bravo JC, Cañas CA. Linear IgA bullous dermatosis associated with systemic lupus erythematosus: a case report. Clin Rheumatol 2008; 27: 391-393.

22. The French Langerhans' Cell Histiocytosis Study Group. A multicentre retrospective survey of Langerhans' cell histiocytosis: 348 cases observed between 1983 and 1993. The French Langerhans' Cell Histiocytosis Study Group. Arch Dis Child 1996; 75: 17-24.

23. Guthery SL, Heubi JE. Liver involvement in childhood histiocytic syndromes. Curr Opin Gastroenterol 2001; 17: 474-478.

24. Al Salloom AA, Almalki ST, Almana H, Burdelski M. Diabetes insipidus and sclerosing cholangitis in a child may be a clue to the diagnosis of Langerhans' cell histiocytosis: a case report. Int J Health Sci (Qassim) 2013; 7: 248-251.

25. Sabib M, Ettair S, Erreimi N, Mouane N. Cholangite sclérosante révélatrice d'une histiocytose langerhansienne chez un nourrisson de 15 mois. Arch Pédiatrie 2011; 18: 974-978.

26. Rhouma FB, Messai H, Hsouna S, et al. History of settlement of villages from Central Tunisia by studying families sharing a common founder Glycogenosis type III mutation. Mitochondrial DNA A DNA Mapp Seq Anal 2016; 27: 3194-3198. 